\title{
THE $U(1)$-INVARIANT FIELD THEORIES WITH NORMAL FIELD OPERATORS
}

\author{
PIOTR ŚNIADY AND MARCIN ZYGMUNT
}

\begin{abstract}
In this paper we consider a very general $U(1)$-invariant field theory such that a field operator commutes with its adjoint, what corresponds to a theory of a charged bosonic particle. We show that from such an invariance follows the existence of particles and antiparticles associated to the same physical state. The field operator always turns out to be a sum of a particle creation and an antiparticle annihilation operators. We study in detail the case when creation and annihilation operators factorize and show that such operators are closely related to $q$-deformed commutation relations.

PACS: $11.10 .-\mathrm{Z}$
\end{abstract}

\section{INTRODUCTION}

In quantum field theory there are today many general theorems which give limitations to possible field theories, e.g. the CPT theorem, the spin and statistics theorem [1].

In this paper we investigate the implications of the $U(1)$-invariance of a field theory. Our assumptions will be very limited, namely we consider a normal field operator $\Phi$ (an operator which commutes with its adjoint) such that the vacuum state is $U(1)$-invariant on the algebra generated by $\Phi$ and $\Phi^{*}$. Particularly we make no assumptions concerning the structure of the spacetime.

We show that from such an invariance follows the existence of particles and antiparticles associated to the same physical state. The field operator turns out to be a sum of a particle creation and an antiparticle annihilation operators. We study in details the case when creation and annihilation operators factorize and show that such operators are closely related to $q$-deformed commutation relations.

Our method bases on an analysis of orthogonal polynomials in two variables [2, 3 .

\section{U(1)-INVARIANT FIELD THEORIES}

We consider a field theory with a Hilbert space $\mathcal{H}$ and a field operator $\Phi: \mathcal{H} \rightarrow \mathcal{H}$. We assume that there is a state $\phi: \mathcal{A} \rightarrow \mathbf{C}$ (where $\mathcal{A}$ is an algebra generated by operators $\Phi$ and $\Phi^{*}$ ) which is $U(1)$-invariant, i.e. if in any expression in $\mathcal{A}$ we replace operators $\Phi$ by $e^{i s} \Phi$ and $\Phi^{*}$ by $e^{-i s} \Phi^{*}$, the value of the state should not change for any $s \in \mathbf{R}$.

Usually the state is simply a vacuum expectation: $\phi(S)=\langle\Omega \mid S \Omega\rangle$, where $\Omega$ denotes the vacuum. In this case the $U(1)$-invariance of a state means that the vacuum is $U(1)$-invariant as well, so the $U(1)$-symmetry of the theory remains unbroken.

Assume that $\Phi$ is a normal operator, i.e. $\Phi \Phi^{*}=\Phi^{*} \Phi$. This assumption is usually fulfilled in the theories of bosonic particles 跑. 
By standard methods of functional analysis we can assign to $\Phi$ a measure $\mu$ with support on the complex plane $\mathbf{C}$ such that

$$
\phi\left(\Phi^{k} \Phi^{* l}\right)=\int_{\mathbf{C}} z^{k} \bar{z}^{l} d \mu(z) .
$$

The $U(1)$-invariance of the state $\phi$ implies that the measure $\mu$ is rotation invariant.

Let us consider polynomials on the complex plane of two variables: $z$ and $\bar{z}$. The following theorem proven by Zygmunt 20 holds:

Theorem 1. Let polynomials $P_{k, l}(z, \bar{z})$ form a system of orthonormal polynomials given by Gram-Schmidt procedure applied to the sequence

$$
1, z, \bar{z}, z^{2}, z \bar{z}, \bar{z}^{2}, \ldots, z^{n}, z^{n-1} \bar{z}, \ldots, \bar{z}^{n}, \ldots
$$

with respect to the rotation invariant measure $\mu$ on $\mathbf{C}$ (the polynomial $P_{k, l}$ has the leading term proportional to $z^{k} \bar{z}^{l}$ ).

For all nonnegative integers $k, l$ they fulfill the following recurrence relations:

$$
\begin{aligned}
& z P_{k, l}=\alpha_{k, l} P_{k+1, l}+\alpha_{l-1, k} P_{k, l-1}, \\
& \bar{z} P_{k, l}=\alpha_{l, k} P_{k, l+1}+\alpha_{k-1, l} P_{k-1, l} .
\end{aligned}
$$

The coefficients $\alpha_{k, l}$ are positive for $k, l \geq 0$ and $\alpha_{-1, l}=0$. Furthermore for all nonnegative integers $k, l$ they fulfill the following relations:

$$
\begin{gathered}
\alpha_{k, l} \alpha_{l, k+1}=\alpha_{l, k} \alpha_{k, l+1}, \\
\alpha_{k, l}^{2}+\alpha_{l-1, k}^{2}=\alpha_{l, k}^{2}+\alpha_{k-1, l}^{2} .
\end{gathered}
$$

Using the notation of this theorem let us denote

$$
\psi_{k, l}=P_{k, l}\left(\Phi, \Phi^{*}\right) \Omega \in \mathcal{H}
$$

We have

$$
\begin{gathered}
\left\langle\psi_{k, l} \mid \psi_{m, n}\right\rangle=\int_{\mathbf{C}} \overline{P_{k, l}} P_{m, n} d \mu(z)=\delta_{k, m} \delta_{l, n}, \\
\Phi \psi_{k, l}=\alpha_{k, l} \psi_{k+1, l}+\alpha_{l-1, k} \psi_{k, l-1}, \\
\Phi^{*} \psi_{k, l}=\alpha_{l, k} \psi_{k, l+1}+\alpha_{k-1, l} \psi_{k-1, l} .
\end{gathered}
$$

We see that $\psi_{k, l}$ is a family of orthonormal vectors in $\mathcal{H}$ indexed by a pair nonnegative numbers. We can think that the index $k$ represents the number of particles and the index $l$ the number of antiparticles which are in the physical state corresponding to the operator $\Phi$.

Equations (4) and (5) tell us that the operator $\Phi=K^{*}+\Lambda$ is a sum of two operators: $K^{*}$ - a creator of a particle and $\Lambda$-an annihilator of an antiparticle, while $\Phi^{*}=K+\Lambda^{*}$ is a sum of a annihilator of a particle and a creator of an antiparticle. 
THE $U(1)$-INVARIANT FIELD THEORIES WITH NORMAL FIELD OPERATORS

\section{FACTORIZABLE CREATION AND ANNIHILATION OPERATORS}

It natural to restrict our considerations to the case when each of the operators $K, K^{*}, \Lambda, \Lambda^{*}$ is a product of two operators, each depending either on the number of particles or the number of antiparticles.

This means that we are solving the equation $\alpha_{k, l}=f_{k} g_{l}$. From the equation (1) it follows that

$$
g_{l} g_{k+1}=g_{k} g_{l+1}
$$

so $\left(g_{i}\right)$ form a geometric sequence. We may take it in the form $g_{i}=q^{i}$ since the constant factor can be moved to the $\left(f_{i}\right)$ sequence. From the assumption $\alpha_{k, l}>0$ follows that $q>0$.

Now the equation (2) takes the form

$$
\left[\left(f_{k}\right)^{2}-\left(f_{k-1}\right)^{2}\right] q^{-2 k}=\left[\left(f_{l}\right)^{2}-\left(f_{l-1}\right)^{2}\right] q^{-2 l}
$$

therefore there exists a constant $c$ such that

$$
\left[\left(f_{k}\right)^{2}-\left(f_{k-1}\right)^{2}\right] q^{-2 k}=c^{2}
$$

from which follows that

$$
\alpha_{k, l}=c \sqrt{1+q^{2}+\cdots+q^{2 k}} q^{l}=\left\{\begin{array}{cc}
c \sqrt{\frac{1-q^{2 k+2}}{1-q^{2}}} q^{l} & \text { for } q \neq 1 \\
c \sqrt{k+1} & \text { for } q=1
\end{array}\right.
$$

We see that the case of $q=1$ corresponds to $\Phi$ being equal (up to a constant) to a sum of a classical bosonic particle creator and antiparticle annihilator.

In the general case we have $K=c A_{k} q^{N_{l}}$ and $\Lambda=c A_{l} q^{N_{k}}$ where $N_{k}, N_{l}$ denote the number of particles or number antiparticles operators respectively and $A_{k}$, $A_{l}$ denote the particle and antiparticls annihilation operators, $A_{k}^{*}, A_{l}^{*}$ denote the particle and antiparticle creation operators.

Operators concerning particles and antiparticles commute:

$$
\begin{aligned}
& A_{k} A_{l}=A_{l} A_{k}, \\
& A_{k}^{*} A_{l}^{*}=A_{l}^{*} A_{k}^{*}, \\
& A_{k}^{*} A_{l}=A_{l} A_{k}^{*} .
\end{aligned}
$$

Creation and annihilation operators fulfill the $q^{2}$-deformed commutation relation [5]:

$$
\begin{aligned}
& A_{k} A_{k}^{*}-q^{2} A_{k}^{*} A_{k}=1 \\
& A_{l} A_{l}^{*}-q^{2} A_{l}^{*} A_{l}=1
\end{aligned}
$$

\section{REFERENCES}

[1] R.F. Streater, A.S. Wightman, PCT, spin and statistics and all that, New York-Amsterdam: W.A. Benjamin (1964)

[2] M.J. Zygmunt, Recurrence formula for polynomials of two variables, orthogonal with respect to rotation invariant measures, Constr. Approx. 15, 301-309 (1999)

[3] P.K. Suetin, Ortogonal'nyje mnogochleny po dvum peremennym, Moskva: Nauka (1988)

[4] N.N. Boglyubov, D.V. Shirkov, Vvedenie v teoriu kvantovannych polej, Moskva: Nauka 1976

[5] U. Frisch, R. Bourret, Parastochastics, J. Math. Phys. 11, 364-390 (1970) 
Institute of Mathematics, University of Wroceaw, Pl. Grunwaldzki 2/4, 50-384 Wroceaw, POLAND, EMAIL: PSNIAMATH.UNI.WROC.PL

Institute of Mathematics, Polish Academy of Sciences, Ul. Śniadeckich 8, 00-950 WarszaWA, POLAND, EMAIL: ZYGMUNTIMPAN.GOV.PL 